C2017, Elsevier. Licensed under the Creative Commons Attribution-NonCommercialNoDerivatives 4.0 International http://creativecommons.org/about/downloads 


\title{
Ignition of Lean and Stoichiometric Air-Propane Mixture with a Subcritical Microwave Streamer Discharge
}

\author{
M.P. Bulat ${ }^{1}$, P.V. Bulat ${ }^{1,2, *}$, P.V. Denissenko ${ }^{3}$, I.I. Esakov ${ }^{4}$, L.P. Grachev ${ }^{4}$, \\ K.N. Volkov ${ }^{5}$, I.A. Volobuev ${ }^{2}$ \\ ${ }^{1}$ Baltic State Technical University, St Petersburg, Russia \\ ${ }^{2}$ ITMO University, 197101, St Petersburg, Russia \\ ${ }^{3}$ University of Warwick, Coventry, United Kingdom \\ ${ }^{4}$ Moscow Radiotechnical Institute of Russian Academy of Sciences, Moscow, Russia \\ ${ }^{5}$ Kingston University, London, United Kingdom
}

\begin{abstract}
Pulse detonation engines are considered to be one of effective propulsion systems for future space missions. Significant efforts are being spent on acceleration of fuel combustion and rising is efficiency. Existing studies have mainly focused on optimizing fuel injection and mixing, repetitive initiation of detonation and integration of detonation tubes with fuel inlets. Understanding of streamer propagation mechanism is of essential importance for the studies of electrical breakdown phenomena and their related applications. In this study, a subcritical microwave streamer discharge is used to initiate ignition of air-fuel mixtures. Ignition of lean fuel mixtures by a streamer has been demonstrated at atmospheric pressure. The speed of streamer-initiated combustion has been shown to be higher compared to that initiated by a spark. The combustion efficiency has also been shown to be higher when using the microwave streamer ignition.
\end{abstract}

\section{Keywords}

Flight safety; Microwave discharge; Streamer discharge; Energy release; Plasma; Ignition; Combustion; Detonation.

\section{Introduction}

Pulse detonation engines (PDE) are unsteady propulsive devices in which the combustion chamber is periodically filled with a reactive gas mixture, a detonation is initiated, the detonation wave propagates through the chamber, and the combustion products are exhausted. The high pressures and resultant momentum flux out of the chamber generate thrust. It suffers, however, from the lack of an appropriate ignition system designed especially for this propulsion technique.

The ignition system has always posed problems in commercial applications. Many experimental, theoretical and numerical studies have been performed for the past years, and various ignition systems (e.g., electric discharge, microwave discharge, laser radiation) have been tested. The propulsive performance of air-breathing PDEs has been theoretically

\footnotetext{
*Corresponding author: pavelbulat@mail.ru
} 
and numerically studied over a wide range of system configurations, operating parameters and flight conditions $[1,2]$. It has been suggested that discharges which create the quickest expanding high temperature region, or discharges which occupy a large volume, are optimal for ignition because they can most rapidly and reliably bring the radius of the ignition kernel to its critical value for transition into a self-propagating flame [3].

In conventional air-fuel mixtures, combustion waves are associated with gas heating, so their distribution is determined by heat transfer processes, which even in the presence of strong turbulence can not give the necessary speed wave propagation. The idea of using plasma methods of fuel ignition is based on non-equilibrium generation of chemically active particles, accelerating the combustion process.

Microwave discharges are widely used for generation of quasi-equilibrium and nonequilibrium plasma. Microwave plasma can be generated at pressures from $10^{-5}$ torr up to atmospheric pressure in the pulse and continuum wave regimes at incident powers ranged between several watts and hundreds of kilowatts. The methods of generation of chemically active discharge plasma, the mechanisms of ignition and combustion controlled by non-equilibrium plasma are discussed in [4].

The study [5] investigates the minimum energy necessary to ignite a laminar premixed methane-air mixture experimentally. A parametrical study is performed to characterize the effects of the flow velocity, equivalence ratio and lens focal length on the minimum energy required for ignition.

A nanosecond pulsed discharge located between two fuel jets was used to ignite and hold jet flames in supersonic crossflow, without the use of additional devices (e.g., cavities or backsteps) for flame holding [6]. Ignition in air-methane mixtures has been achieved using low energy seed laser pulses and an overlapping subcritical microwave pulse [7]. It is shown that an extremely weak ionization by a laser localizes the microwave energy deposition and leads to rapid heating, high temperatures and ignition. Multiple simultaneous localized regions of ignition are also achieved using the same microwave pulse.

Preliminary experiments and theory estimations have shown that propane injected into high-speed airflow can be successfully ignited by microwave subcritical attached discharge [8]. This type of discharge is arising at the sharp tip of a passive vibrator placed into microwave radiation. The important property of the attached discharge is its ability to exist in microwave field of a small level. The study [9] is devoted to particular mechanisms of the plasma effect on high-speed combustion.

Mechanisms for the acceleration of combustion under the action of a non-equilibrium plasma are actively discussed including the generation of atomic oxygen and other chemical radicals [10], development of molecules of singlet delta-oxygen [11,12], chain ion-molecular reactions with intermediate radicals [13]. The possibility of a non-equilibrium electric discharge is experimentally demonstrated under conditions of heat engines [14-17].

There are different stages of development of microwave discharge including diffuse, ionization-overheating, streamer, resonant and cumulative stages [18]. The main physical factors which determine the properties of a discharge in each of the stages do not depend on the kind of gas. However, while the general picture of the development of discharges in different gases is qualitatively consistent, its specific details may differ. An atmospheric pressure pulsed microwave discharge on the surface of an insulating plate and between two insulating films is considered in $[19,20]$.

The study focuses on investigation of possibilities of the use of microwave radiation to initiate combustion and detonation of air-fuel mixtures. The results of experimental and computational studies related combustion and detonation of air-propane mixture are presented. To initiate the combustion and detonation, the deep subcritical streamer discharge 
is used. The discharge is formed by a field with the intensity smaller than the minimum pulse intensity leading to the gas breakdown. An acceleration of combustion and a uniform temperature front are obtained, and the possibility of combustion of lean fuel is confirmed.

\section{Types of microwave discharges}

At the pressure of a mixture, exceeding 0.1 atmospheric pressure, microwave discharges get a streamer nature. It is a continuous sequence of the electrodeless spark channels, irregularly inducing one another and carried away by a stream from area of occurrence.

High- and medium-pressure discharges excited by quasi-optical microwave beams in air are classified in [20] using experimental data. Diffuse, streamer, overcritical, subcritical and deep subcritical discharges are distinguished. The domains of existence of each type of discharge are determined as functions of the air pressure and field strength for two particular electromagnetic wavelengths. As the wavelength decreases, the domain of existence of an subcritical streamer discharge with a developed spatial structure shifts toward higher pressures and higher field strengths.

The domains of existence of various types of microwave discharge are shown in the Figure 1 , where $E_{0}$ is the electric field of the microwave beam in the discharge region and $E_{c}$ is the critical breakdown electric field. They are designated by Roman numerals I-V, respectively. These domains are determined by analyzing a series of time-integrated photographs of discharges operating at different initial field strengths and different air pressures.

Line 1 in the Figure 1 corresponds to the dependence $E_{c}(p)$. Above this line, selfsustained air breakdown occurs, whereas below it, air breakdown has to be initiated. Line 2 , which corresponds to the experimentally determined threshold pressure $p_{\text {thres }}$, separates the domains of existence of diffuse and streamer discharges, which occur to the left and to the right of this line, respectively. Line 3 corresponds to the experimentally determined field $E_{\text {thres }}(p)$. In the pressure range $p>p_{\text {thres }}$, it separates subcritical $\left(E_{0}<E_{c}\right)$ streamer discharges with a developed spatial structure that are capable of detaching from the initiator and deep subcritical $\left(E_{0} \ll E_{c}\right)$ streamer discharges, whose plasma channels are attached to the initiator. Line 2 , which corresponds to $p_{\text {thres }}$, is conventional. In experiments, there is an air pressure range (the hatched region in the Figure 1) in which it is difficult to distinguish between diffuse and streamer discharges. Over the entire range of air pressures, there is a narrow transition region below line $1\left(E_{0}<E_{c}\right)$ in which weak reflection of microwave radiation from an initiated subcritical discharge can lead to self-sustained air breakdown in the microwave beam in front of this discharge.

Self-sustained breakdown could occur only at air pressures lower than $p=100$ torr. At pressures below several tens of torrs, the discharge operated in the diffuse mode. At pressures exceeding a certain threshold value $p_{\text {thres }}$, a diffuse discharge transformed into a streamer one.

The spatial structure and properties of a overcritical $\left(E_{0}>E_{c}\right)$ diffuse discharge operating at an air pressure of $p<p_{\text {thres }}$ are primarily determined by the field-ionization processes. Gas ionization in the discharge region is governed by the mutual dynamic influence of the initial field and the field generated in the plasma produced. The discharge structure depends on the air pressure. At low pressures (typically, at $p<10$ torr), the discharge region with $E_{0} \geqslant E_{c}$ was nearly uniformly filled with a plasma. As the pressure increased, the plasma broke into separate diffuse plasmoids. An overcritical diffuse microwave discharge consumed almost no microwave field energy, and the air in the discharge region was heated by no more than several tens of degrees. 


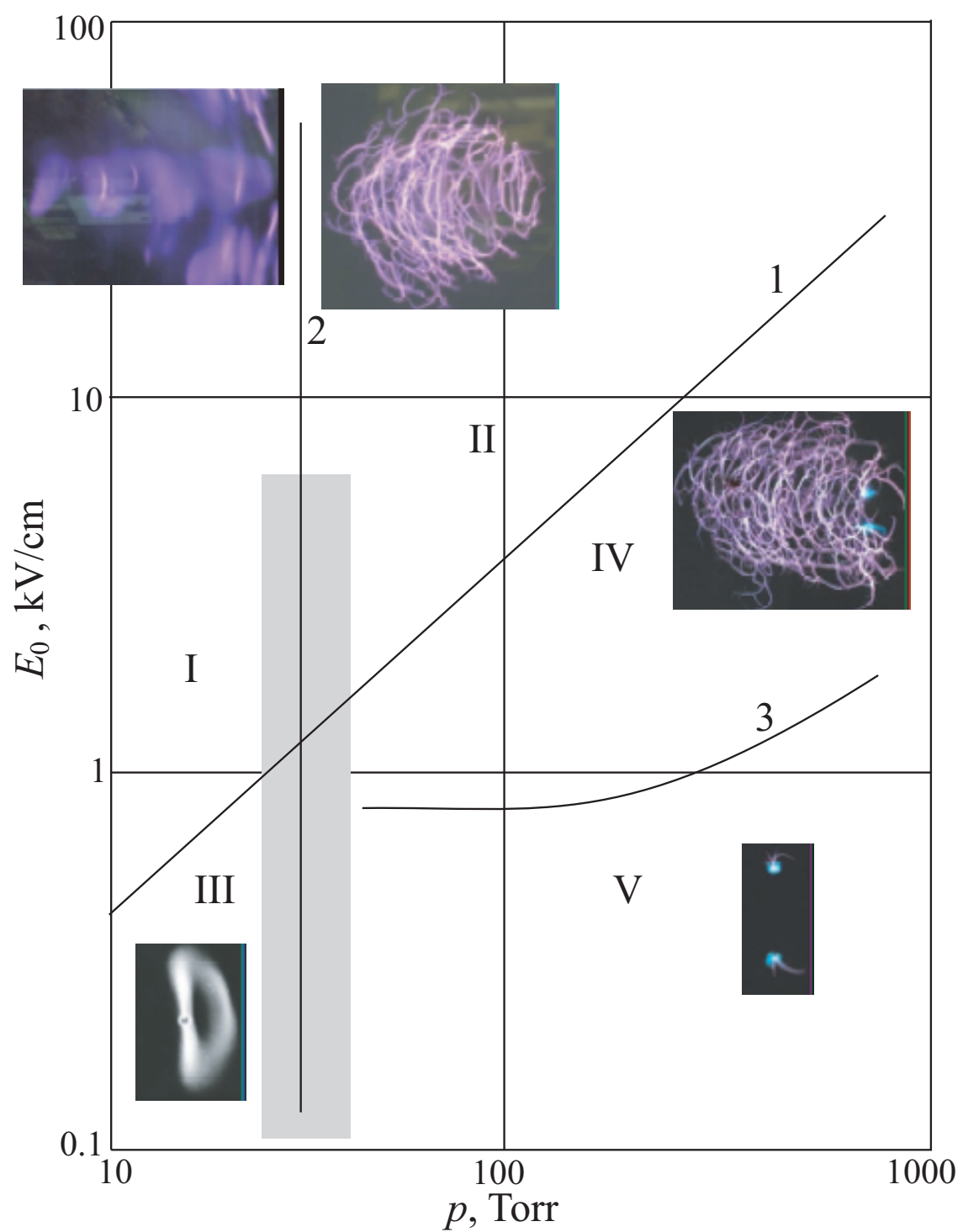

Figure 1. Domains of existence of different types of microwave discharges for $\lambda=8.9 \mathrm{~cm}$ in air [20]

The typical overcritical $\left(E_{0}>E_{c}\right)$ streamer discharge operating at a pressure of $p>$ $p_{\text {thres }}$ discharge presents a rather complicated system of thin plasma channels. It originates against the free-electron background and then develops as a system of growing and branching streamer channels. The ionization of air in the channels is substantially affected by the ionization-heating process, whereas their growth and branching are determined by the magnitude and distribution of the field at the channel heads. The overcritical streamer discharge completely absorbs microwave energy and the gas temperature in the resonant plasma channels increases to $1000 \mathrm{~K}$ and more.

A discharge in a quasi-optical microwave beam can be excited even in an subcritical field $\left(E_{0}<E_{c}\right)$. In order to achieve gas breakdown under these conditions, special measures should be undertaken (breakdown should be initiated). Similarly to discharges in an overcritical field, subcritical initiated microwave discharges can operate in either the diffuse or the streamer mode, depending on the air pressure. When the air pressure exceeds the threshold value, a diffuse initiated discharge also transforms into a streamer one. 


\section{Experimental technique}

Schematic of the experimental setup is shown in the Figure 2. The test rig includes a microwave generator that produces electromagnetic waves with a frequency $f \sim 3.4 \mathrm{GHz}$ which corresponds to the wavelength $\lambda=8.9 \mathrm{~cm}$. Duration of the microwave pulse is 40 $\mu \mathrm{s}$ and the power can be varied in the range $10^{2}-10^{6} \mathrm{~W}$. After leaving the waveguide, the microwave beam is expanded to the diameter of $60 \mathrm{~cm}$ by a dielectric lens and then focussed by a mirror. The focus area has the diameter of approximately $10 \mathrm{~cm}$ and the characteristic length of $15 \mathrm{~cm}$.

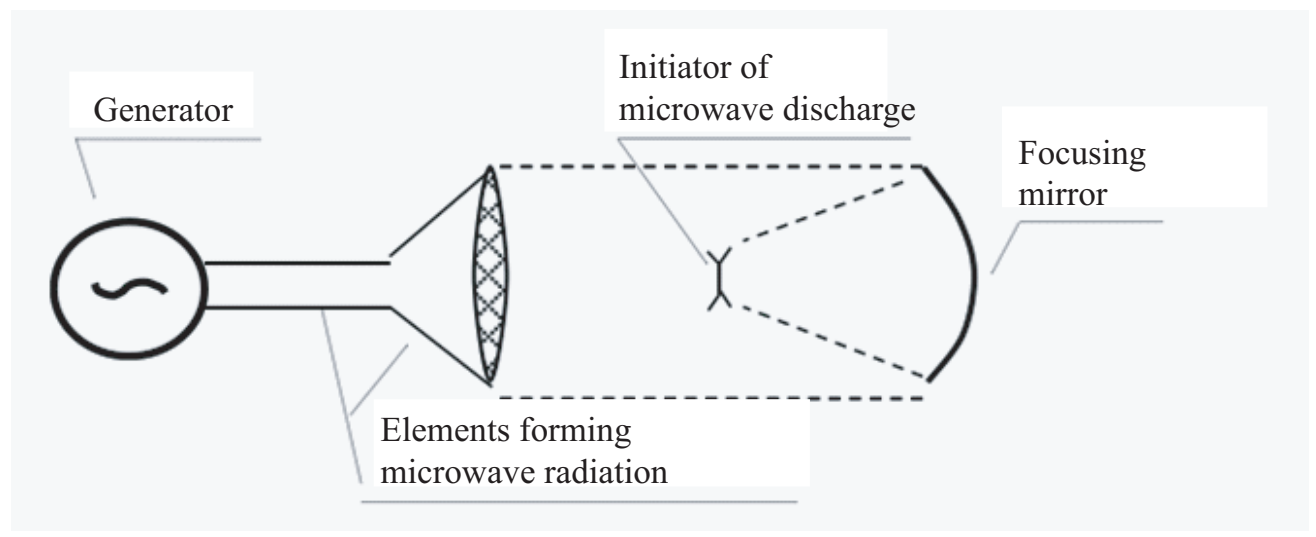

Figure 2. Layout of the experimental test rig

In the focus, a half-wave length wire is placed aligned with the electric field polarization to initiate the streamer discharge. It allows initiating the streamer microwave discharge at the atmospheric pressure and the electric field magnitude hundreds of times smaller than the breakdown value.

A rubber balloon with a diameter of $15 \mathrm{~cm}$, filled with air-propane mixture is attached directly to the initiator (Figure 3). A streamer discharge passes inside the balloon, igniting the mixture. Air excess coefficient, $\alpha$, varies from 0.25 to $1.5(\alpha=1$ corresponds to the stoichiometric air to fuel ratio, which is 15.6:1 for the air-propane mixture). To study the effect of microwave field intensity on the type of generated discharge and the nature of combustion, the initiator is placed in the focus of the mirror and then shifted along the axis.

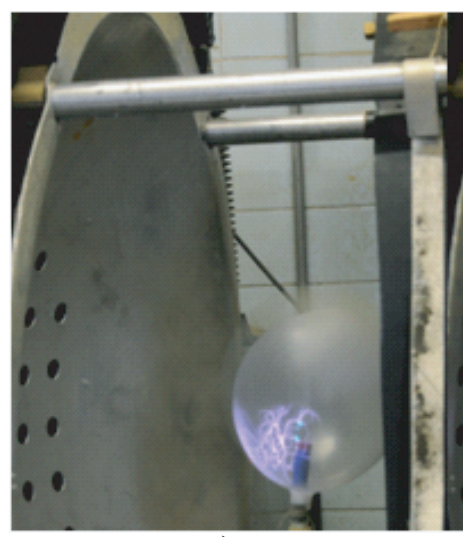

a)

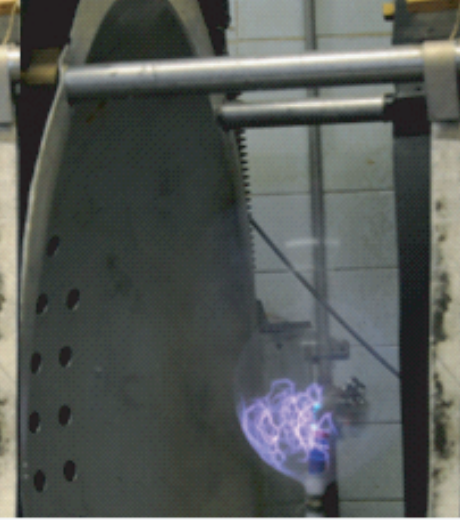

b)

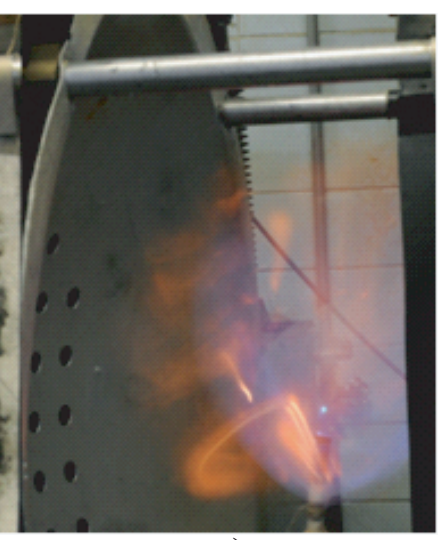

c)

Figure 3. Combustion of a balloon filled with air-propane mixture by a streamer discharge

Development of the combustion process is compared for cases of streamer and spark ignition. Development of the streamer and the spread of the flame front has been recorded 
using a monochrome high-speed camera Phantom v.2511 with the frame rate of up to 750,000 frames/s and the color Nikon1 J2 camera at a frame rate of 1200 frames/s. The speed of the flame front propagation and the induction time from the moment of the discharge to the beginning of combustion reaction have been measured.

The results of measurements of the electric field strength are shown in the Figure 4. In the tests, power and energy of microwave beam remain the same. To vary energy of the microwave discharge, the discharge initiator shifts relative to the focus, and the electric field strength and energy release are found from the graph.

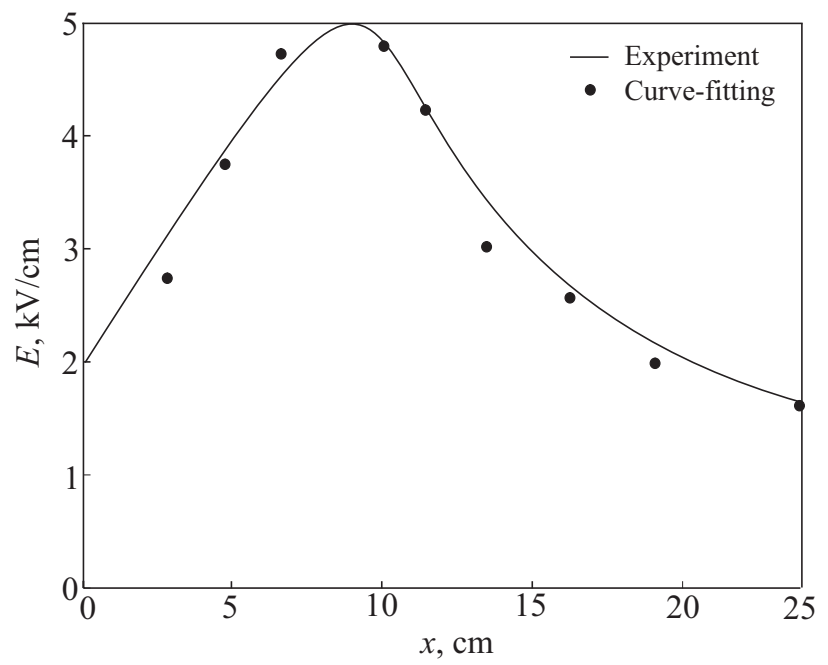

Figure 4. Electric field strength as a function of distance from the mirror edge based on measurements (symbols) and curve-fitting (line)

\section{Streamer discharge}

The streamer is present in many applications and physical phenomena, involving numerous time scales and characteristic lengths. One of the main properties of a developed microwave streamer discharge is that it absorbs almost all the electromagnetic energy incident on it. This is a significant difference between microwave streamer discharges and spatially uniform discharges. Another important feature of microwave streamer discharge is that the streamers of which they are comprised, like streamers in a direct current field, grow into a region of the electromagnetic beam where the amplitude of the electric component of the initial field is substantially lower than the critical breakdown amplitude.

In the microwave field of overcritical amplitudes an initial electronic avalanche is arising from a single initial electron. In process of growth of amplitude of an electric field in focus of the beam there is an area in which the field exceeds critical (breakdown) value. Occurrence in this area a free electron causes development of an electronic avalanche.

An estimation of growth speed of subcritical streamer discharge is [8]

$$
v_{S}=3.6 \cdot 10^{5}\left[\lambda_{\mathrm{cm}} \log \left(\frac{30 p_{\text {torr }}}{E_{\mathrm{V} / \mathrm{cm}}}\right)\right]^{-1 / 2}[\mathrm{~cm} / \mathrm{s}],
$$

where $\lambda_{\mathrm{cm}}$ is the wavelength of microwave radiation $[\mathrm{cm}]$. The free electron diffusion coefficient is $D_{e}=10^{5} / p_{\text {torr }}\left[\mathrm{cm}^{2} / \mathrm{s}\right]$, and the critical value of electric field is $E_{c}=30 p_{\text {torr }}$ $[\mathrm{V} / \mathrm{cm}]$. 
The equation (1) shows a weak dependence of speed on subcriticality, an electric field and pressure until the field does not come nearer to critical. The equation (1) gives the values of the growth rate rather close to measured in various experiments [21], that specifies a correct choice of the determining factor of development of subcritical discharge. In the Figure 5, an estimation of the streamer growth rate (solid line) is compared with measurements from [21] for $p=200$ torr and $\lambda=8.9 \mathrm{~cm}$ (bullets). The equation (1) underestimates speed of propagation, though the general course of dependence is reproduced well. Estimation (1) at constant value of subcriticality predicts full independence of speed on pressure and on electric field. This result misses the data of the measurements [21] indicating some growth of speed with growth of pressure, however this divergence does not leave the limits of accuracy of measurements.

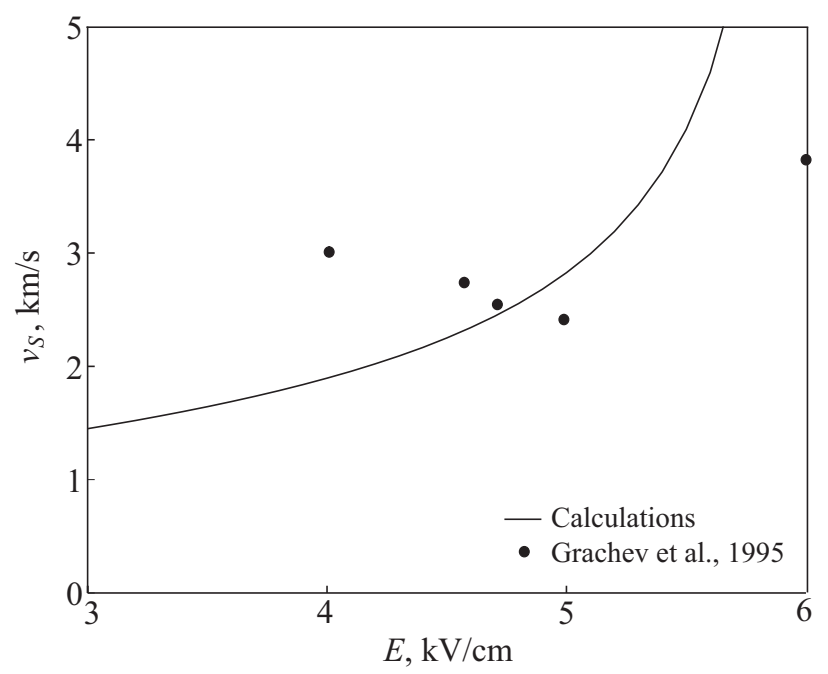

Figure 5. Dependence of speed of subcritical streamer on amplitude of an electric field for $p=200$ torr and $\lambda=8.9 \mathrm{~cm}$

The radius of a head part of the streamer channel is determined by depth of front of ionization

$$
a_{S}=\frac{11.1}{p_{\text {torr }}}\left[\lambda_{\mathrm{cm}} \log \left(\frac{30 p_{\text {torr }}}{E_{\mathrm{V} / \mathrm{cm}}}\right)\right]^{1 / 2}[\mathrm{~cm}] .
$$

Dependence of radius of the channel on pressure of gas is shown in the Figure 6. Typical value of speed of development of the streamer of subcritical discharge lays in a range of units of kilometers per second. For an estimation of its value the equation (1) can be used.

An estimation for volumetric density of the energy, absorbed by a streamer fragment, normalized on initial value of enthalpy, is

$$
q=2.2 \cdot 10^{-8} \frac{\lambda_{\mathrm{cm}}^{3 / 2} p_{\text {torr }} E_{\mathrm{V} / \mathrm{cm}}}{C_{p}}\left[\log \left(\frac{30 p_{\text {torr }}}{E_{\mathrm{V} / \mathrm{cm}}}\right)\right]^{-1 / 2} .
$$

Figure 7 shows that subcritical discharges are capable to lift temperature in the streamer channel many times over, essentially exceeding temperature of ignition of air-propane mixtures [22].

The radius of the channel of attached discharge is limited by radius of the sharp end of the initiator, which is obliged to provide the local increase of an electric field above critical value. For the half-wavelength metal vibrator, oriented along a vector of an electric field of 


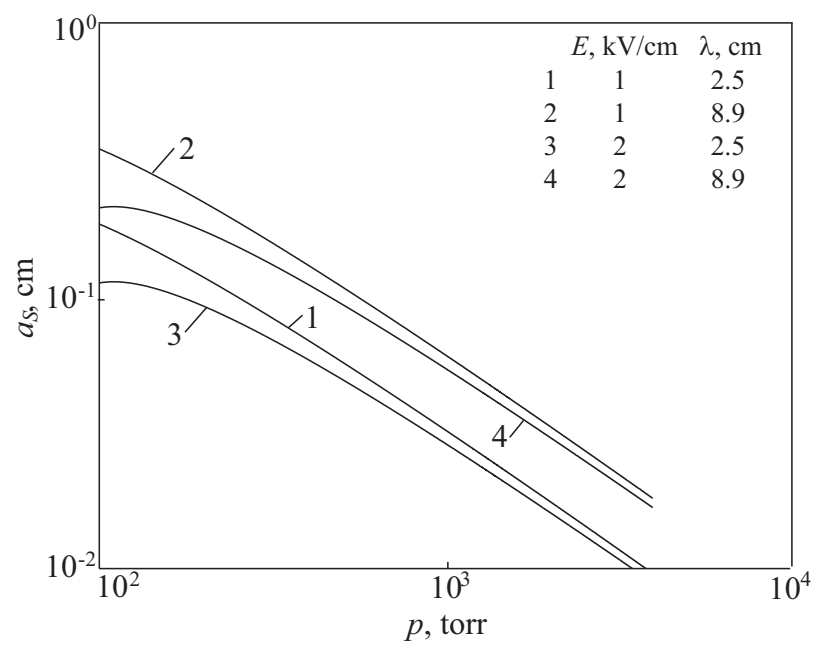

Figure 6. Estimation of radius of the streamer channel of subcritical microwave discharge

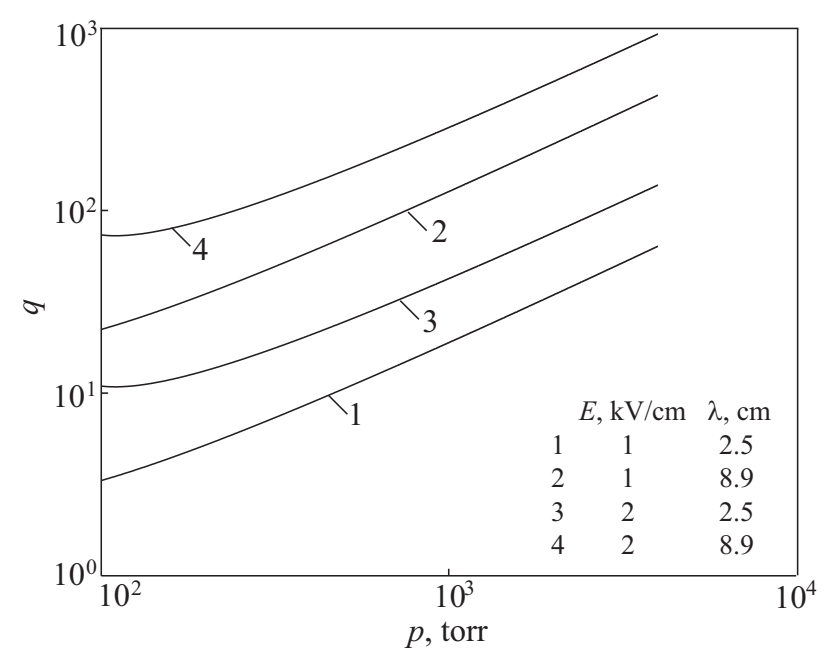

Figure 7. Estimation of specific energy of gas in the streamer channel of subcritical discharge

radiation, the factor of increase of a field is approximately equals to $4 a$, where $a$ is the radius of curvature of the end of the vibrator. The radius of attached discharge is

$$
a_{S}=\frac{\lambda_{\mathrm{cm}}}{4} \frac{E_{0}}{E_{c}} .
$$

Equation (4) is shown in the Figure 8.

Defining a characteristic length size as $a_{S}=\lambda /(2 \pi)$, a condition for the border dividing free and attached subcritical types of discharges can be deduced

$$
\lambda_{\mathrm{cm}}^{1 / 2} E_{\mathrm{V} / \mathrm{cm}}\left[\log \left(\frac{30 p_{\text {torr }}}{E_{\mathrm{V} / \mathrm{cm}}}\right)\right]^{-1 / 2}=2 \cdot 10^{3} .
$$

Solution of equation (5) for fixed wavelengths is compared with dependence of a critical field on pressure of air in the Figure 9. Bullets correspond to the data of measurements from [23]. The general course of dependence on pressure and lengths of a wave quite correspond to observations, that also proves the advanced representations about streamer discharge. 


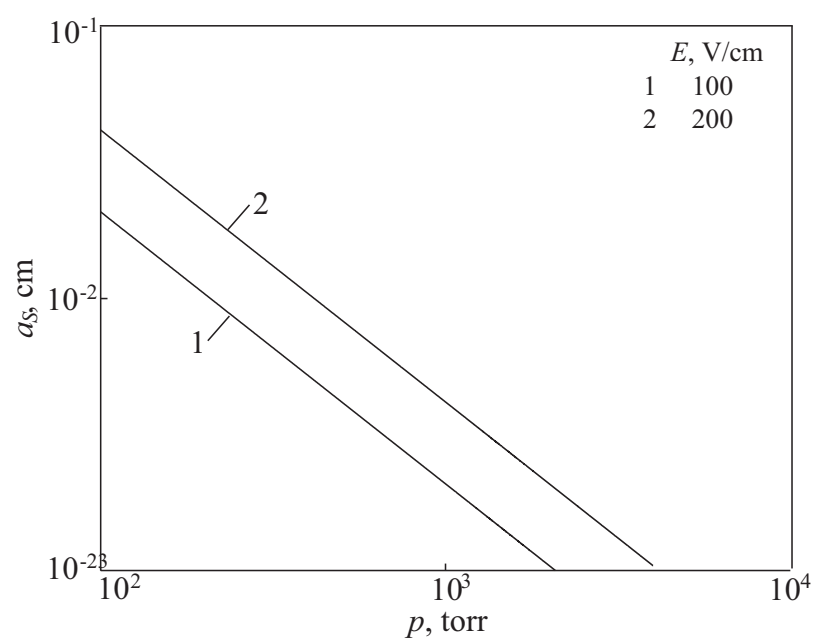

Figure 8. Dependence of radius of the channel of the attached discharge on pressure

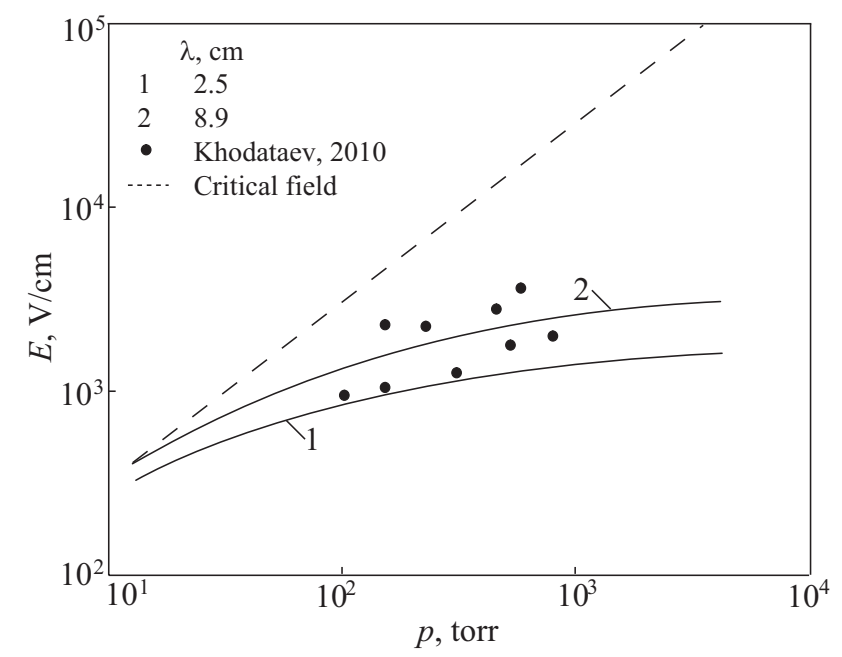

Figure 9. Domains of existence of the main types of streamer discharges for $\lambda=2.5 \mathrm{~cm}$ (line 1) and $\lambda=8.9 \mathrm{~cm}$ (line 2)

\section{$5 \quad$ Numerical analysis}

During the microwave discharge, the convective motion of the plasma is small compared to the characteristic size of the problem (radius of balloon). In the numerical simulations it is assumed that energy is released instantaneously. Instantaneous energy deposition by high-power microwave discharge into a small gas volume generates an explosion. The point explosion problem without backpressure has an analytical solution (Sedov-Taylor problem). Using some assumptions, this analytical solution is derived in the blast wave approximation with spherical, cylindrical and plane symmetry [24].

The microwave generator used in experiments is not powerful enough to consider the discharge as a strong explosion, and Sedov-Taylor model without backpressure is not valid in this case. To study combustion and detonation of air-fuel mixture in the balloon, methods of computational fluid dynamics are applied.

The governing equations describing the problem include continuity equation, momentum conservation equation, energy conservation equation and equation of state of ideal gas. The governing equations are solved numerically with the finite volume method [25]. The explicit Runge-Kutta scheme of the third order is used for time discretization, and MUSCL scheme 
is exploited for space discretization. To calculate the numerical fluxes on the control volume faces, Roe approach is applied.

During combustion, the burned gas composition is generally calculated on the assumption of chemical equilibrium at the given temperature and pressure. Comprehensive detailed kinetic mechanisms have been compiled to fully describe the fundamental chemical processes involved in fuel oxidation. Thermodynamic data for elements, combustion products, and many pollutants are available in a compilation published by the National Bureau of Standards (JANAF Tables).

\section{Results and discussion}

The subcritical streamer discharge is formed by a field with the intensity smaller than the minimum pulse intensity leading to the air breakdown. Streamer discharge ignition of lean fuel mixture with excess oxidant ratio greater than flammability limit under normal conditions is studied.

The experiment with spark initiated combustion of stoichiometric air-propane mixture is conducted first. Balloon is inflated with fuel mixture to a diameter of $15 \mathrm{~cm}$ and it is ignited by a spark plug. A diffusion combustion process occurs shown in the Figure 10. At the place of ignition a bright glowing orange spot, typical for areas with large temperature gradient, remained for a long time, in which nitrogen oxides are formed mostly. The velocity of flame front propagation measured by camera is $1.5-2 \mathrm{~m} / \mathrm{s}$. The flame has a blue-green shade, indicating the presence of yellow lines in the emission spectrum, typical for glowing of soot particles and nitrogen oxide. At the moment when the balloon burst, the ejection of bright yellow turbulent flame occurs, which continues to glow for another $0.1 \mathrm{~s}$. The nature of the flame indicates that the combustion of propane does not occur completely, and the supply of large excess of oxygen causes an increase in temperature and an intensive glow of soot particles and unburned fuel.

A similar experiment is conducted with the initiation of combustion by a streamer discharge. The observed pattern of combustion presented in the Figure 11 is different from the case of the spark ignition. The propagation speed of the flame front is $6-10 \mathrm{~m} / \mathrm{s}$. The balloon burst two times faster than with spark ignition. The flame has smooth purple color, typical for combustion of pure natural gas. Orange spot does not appear. However, the flame front is turbulent, and numerous so-called hot points formed on its fractures of which the detonation develops. They can be seen on the photos as red color areas. After balloon burst, no flame ejection occurs, and there is no afterglow either (the fuel is burned completely).

The ignition by a streamer discharge changes the nature of combustion. The rate of combustion and combustion efficiency increase by times, and no soot is formed.

Experiments with streamer ignition are repeated for $\alpha=0.25,0.33,0.4$ and 0.5 , when the amount of fuel is smaller than flammability limit $(\alpha=0.56)$. At $\alpha=0.5$ ignition occurs, and ignition is not observed at lower $\alpha$. Thus, it is confirmed that by means of streamer discharge it is possible to ignite lean fuel mixture, but only within reasonable limits. Experiments have shown that the combustion of lean mixture is about two times slower than of the rich one, but is clean and the hot spots are not observed. After the balloon bursts, a flame ejection and an afterglow are completely absent.

The attached discharge and the discharge of transition type are both investigated for the ignition of stoichiometric mixture. Combustion initiated by attached discharge is similar to combustion induced by a spark. Flame has a diffusive nature. The propagation velocity of flame front is about 1-2 m/s. However, the combustion efficiency is higher, and the flame 
a)

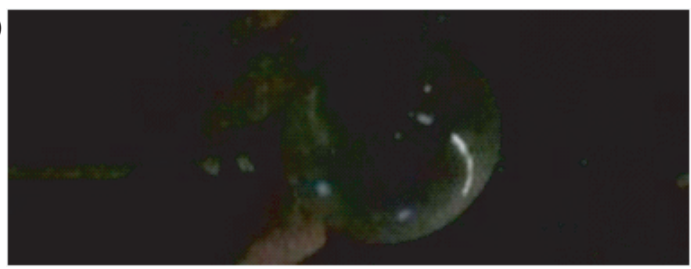

c)

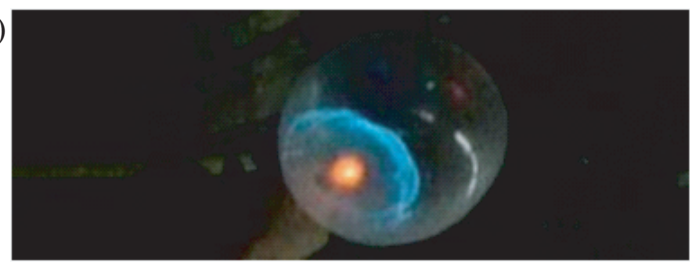

e)

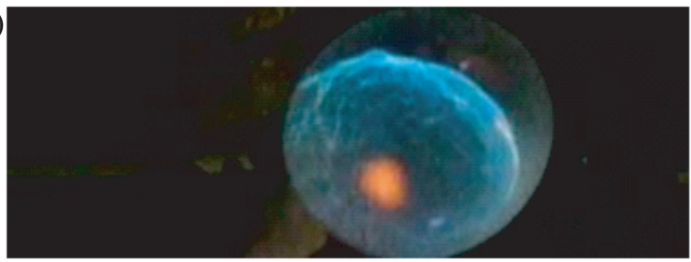

g)

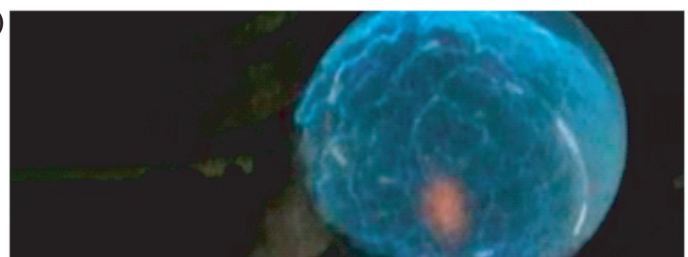

b)

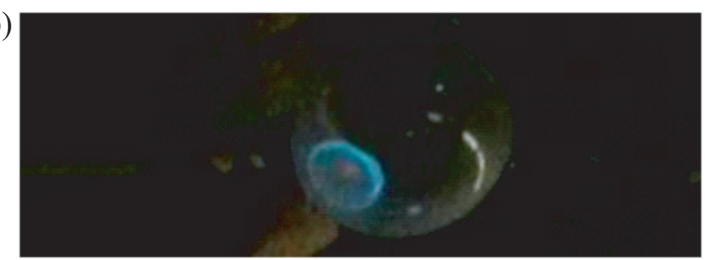

d)
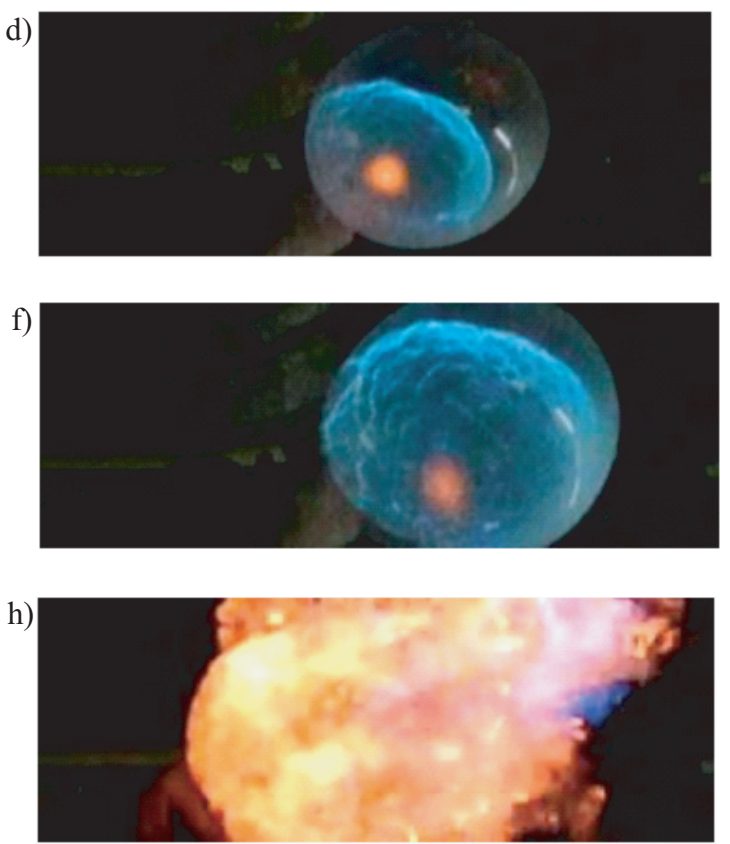

Figure 10. Ignition of a balloon filled with a stoichiometric air-propane mixture with a spark: 0 (a), $18 \mathrm{~ms}$ (b), $36 \mathrm{~ms}$ (c), $54 \mathrm{~ms}$ (d), $72 \mathrm{~ms}$ (e), $90 \mathrm{~ms}$ (f), $108 \mathrm{~ms}$ (g), $126 \mathrm{~ms}$ (h). Initial diameter of the balloon is $15 \mathrm{~cm}$. Front propagation speed is $1.5 \mathrm{~m} / \mathrm{s}$

ejection after the bursting of the balloon is not as bright. The discharge itself is not like a streamer discharge. It looks like a small plasma formation with diffusion structure. Ignition has point nature (occurs at single point), as in spark ignition. The attached discharge does not give significant advantages in comparison with spark ignition, and the ignition mechanism in this case is of the heat type.

Transition discharge, much like streamer discharge, has a developed structure of plasma channels, but the volume they occupy is much smaller. The combustion nature is similar to combustion ignited by streamer discharge. The velocity of flame front propagation is comparable, but the initial ignition amount is much smaller, so the total time of mixture burning is larger, and the combustion efficiency is smaller than for streamer discharge.

The mechanism of ignition of the the mixture is common for both streamer discharge and transition discharge. Differences in combustion process are due to lower volume, in which the transition discharge develops, and to smaller supply energy. Combustion ignited by attached discharge is qualitatively different, and it is similar to spark ignited combustion. The streamer discharge and transition discharge are different from attached diffusion discharge by developed structure of plasma filaments. The different nature of ignition and combustion is determined by a filamentary structure of streamer and transition discharges.

Some experiments are conducted with the frame rate increased to 1,000,000 frames/s. This allows to observe the development of the initial combustion phase in detail. Figure 12 shows a frame sequence of a streamer discharge for the most typical case of combustion at $\alpha=0.5$. In the lower-left corner of the each frame the time to within 1 microsecond is given. With this frame rate the camera could only shoot in black and white regime. The discharge 
a)

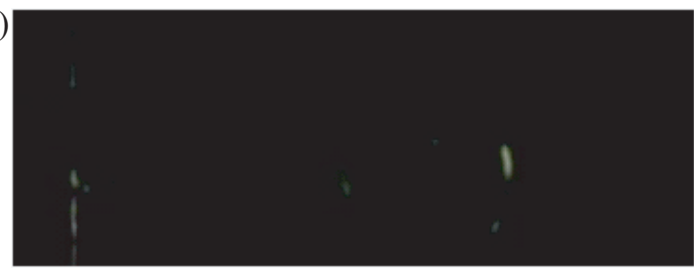

c)

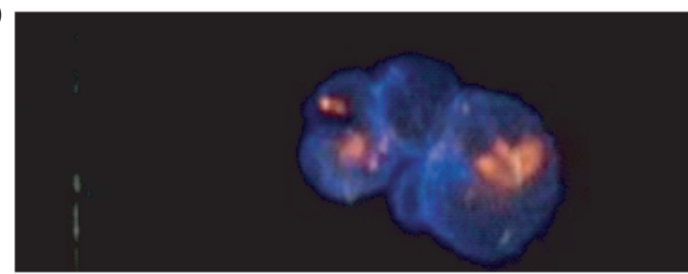

e)

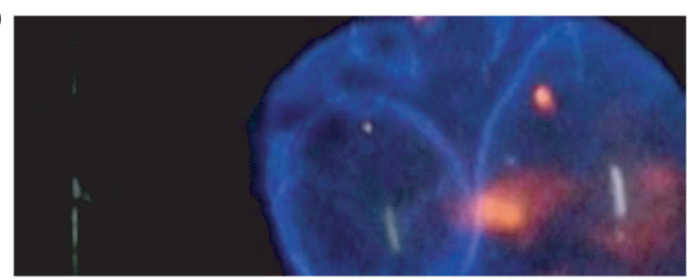

g)

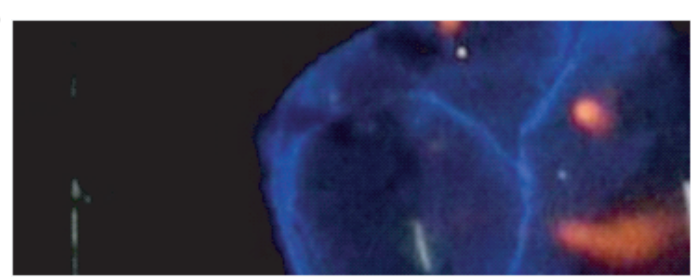

b)

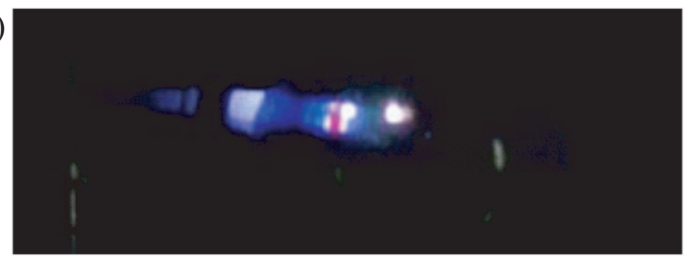

d)
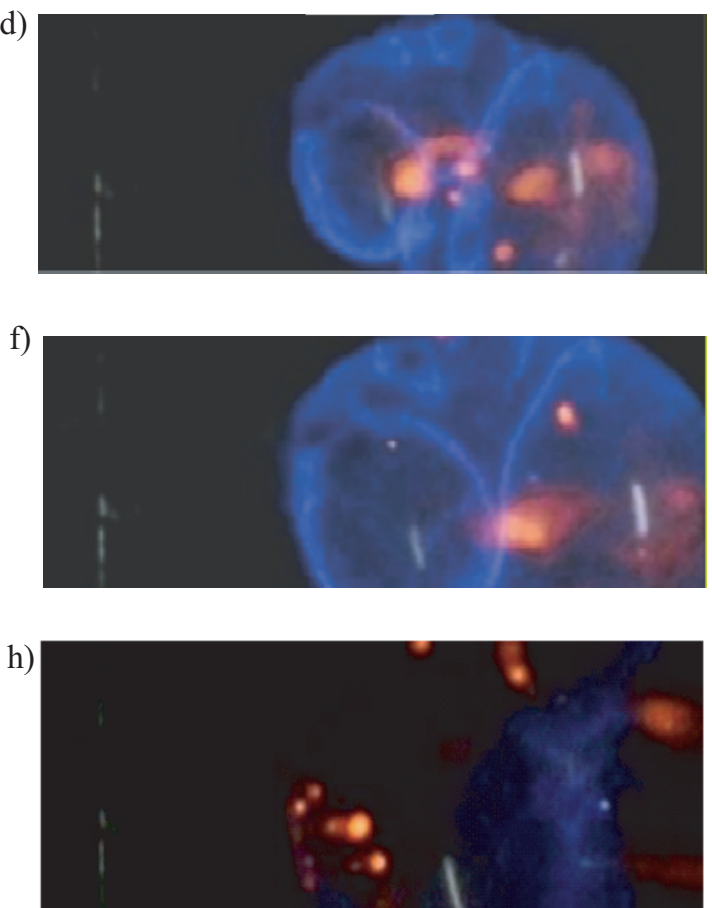

Figure 11. Ignition of a balloon filled with a stoichiometric air-propane mixture with a

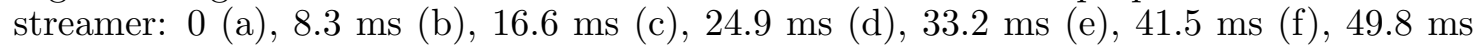
$(\mathrm{g}), 58.1 \mathrm{~ms}(\mathrm{~h})$. Initial diameter of the balloon is $15 \mathrm{~cm}$. Front propagation speed is 7 $\mathrm{m} / \mathrm{s}$

is ignited time under the influence of microwave radiation pulse (Figure 12a). After about 10-15 microseconds a bright flash is accompanied by a glow (Figure 12b), in approximately 40 microseconds more a developed streamer structure is observed (Figure 12c), then the discharge is extinguishing for 50-60 microseconds and a trace is left in its place (Figure 12d).

The experiment is repeated for a transitional discharge. In this case, the discharge occupies a smaller area and, consequentially, the combustion began in a smaller volume. Experiment with attached diffusion discharge shows complete analogy with spark ignition (Figure 13). The discharge is ignited in a form of plasma cloud (Figure 13a). After about 2.3 microseconds (Figure 13b) the ignition of mixture begins (the induction time exactly corresponds to conventional thermal ignition regime). At the place of ignition a bright spot with a large temperature gradient is formed which is visible for about 10 microseconds (Figure 13c). Then, the area of combustion and high temperature occupies the entire volume (Figure 13d).

A number of studies states that the streamer discharge is accompanied by powerful ultraviolet glow, causing an avalanche increase in number of free electrons [23]. The appearance of filamentary plasma channel is associated with developmental of ionization-overheating instability process in the plasma of microwave discharge, which is accompanied by a powerful ultraviolet radiation from the discharge zone. Microwave discharge excites oxygen atoms resulting in generation of powerful ultraviolet radiation, which causes the formation of nonequilibrium cold plasma with an avalanche increase in the number of free electrons. The 

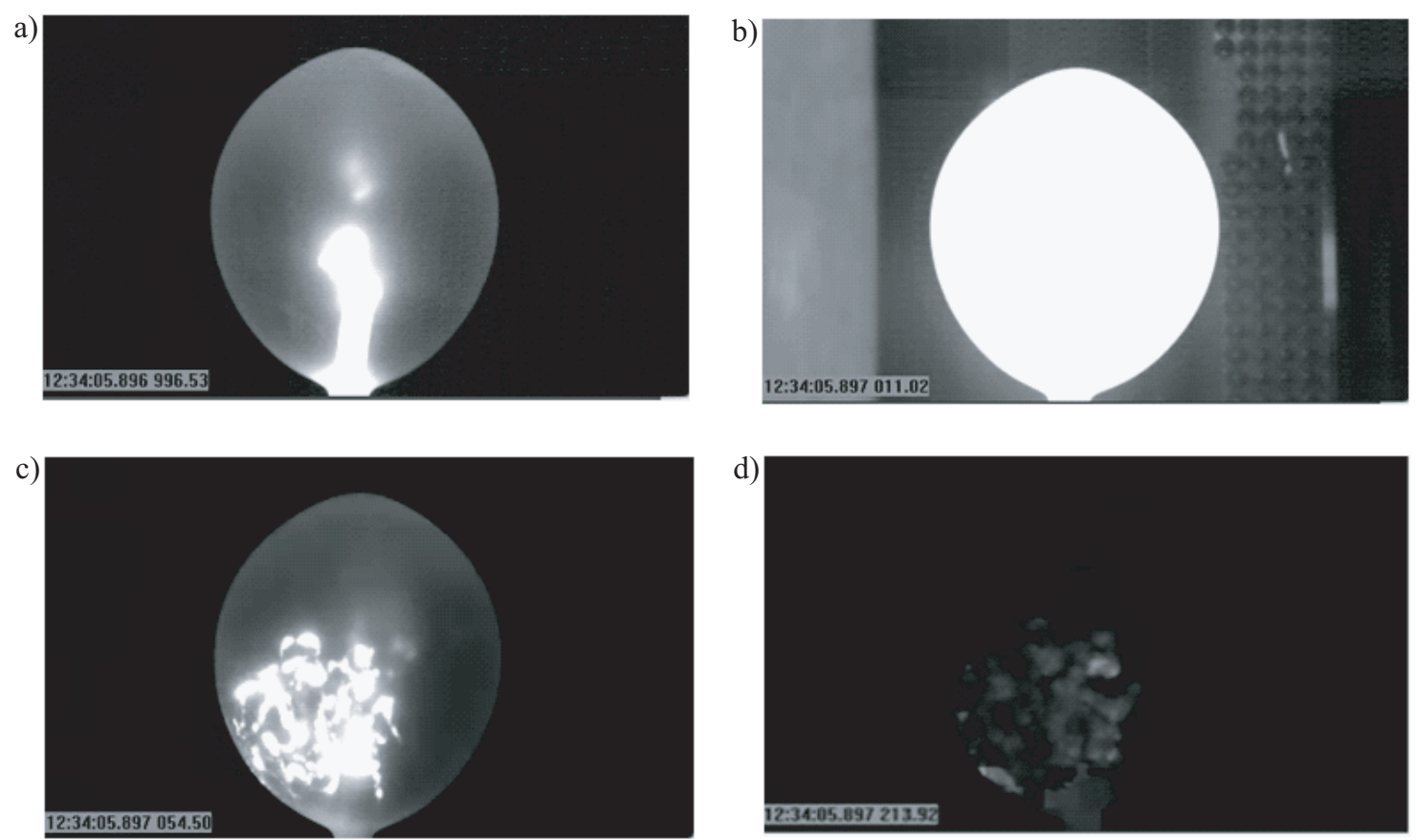

Figure 12. Development of a streamer discharge: discharge ignition (a); bright flash (b); developed streamer structure (c); trace of extinct discharge (d)

experiments show that the streamer discharge propagates at a speed of about $5 \mathrm{~km} / \mathrm{s}$, so the ignition is initiated over the whole volume where the plasma filaments propagate. The temperature of fuel mixture at the point of ignition initiation is not higher than $400 \mathrm{~K}$.

The microwave subcritical discharge causes detonation or combustion depending on composition of the mixture and the radiation power at fixed pulse duration of 40 microseconds. The initiation of detonation is possible not only in the case of a stoichiometric mixture, but in the case of a lean mixture when the equivalent ratio is larger than 0.45 . The dynamics of development of streamer discharge is shown in the Figure 14 (period frames is 5 microseconds, exposition is 2 microseconds), which shows the single-frame scan, obtained by speed cameras.

Initiation of detonation with the use of subcritical microwave discharge, carrying the energy release in a small region with a typical size smaller than $0.1 \mathrm{~cm}$ and using subcritical discharge with a developed system of streamers covering an area with a characteristic size of the order of several centimeters, is studied. In the latter case, the initiation of detonation is carried out simultaneously along the streamer discharge channels. The power output dissipated in the discharge is estimated on top of the measured amplitude of the electric field based on electrodynamic calculations. For a given pulse duration of the microwave radiation and the measured values of the total length of the streamer system, the total energy of detonation is defined. The characteristic values of the total energy at which the detonation of a stoichiometric mixture takes place, is equal to $1.2 \mathrm{~J}$ for attached discharge and $0.2 \mathrm{~J} / \mathrm{cm}$ for streamer discharge. High-speed camera with high temporal resolution (exposure frames and delay between frames is adjustable from 2 to 100 microseconds for an arbitrary delay from the beginning of the process) and digital video camera are used.

When interpreting the high-speed camera data, the dynamics of the motion of the balloon shell is an important issue. In the case of combustion of a mixture, the energy release in a balloon occurs slowly, so that the balloon is inflated, maintaining a pressure which is close to the external pressure (atmospheric in the experimental conditions). However, in the case of detonation, the process takes place much faster. 


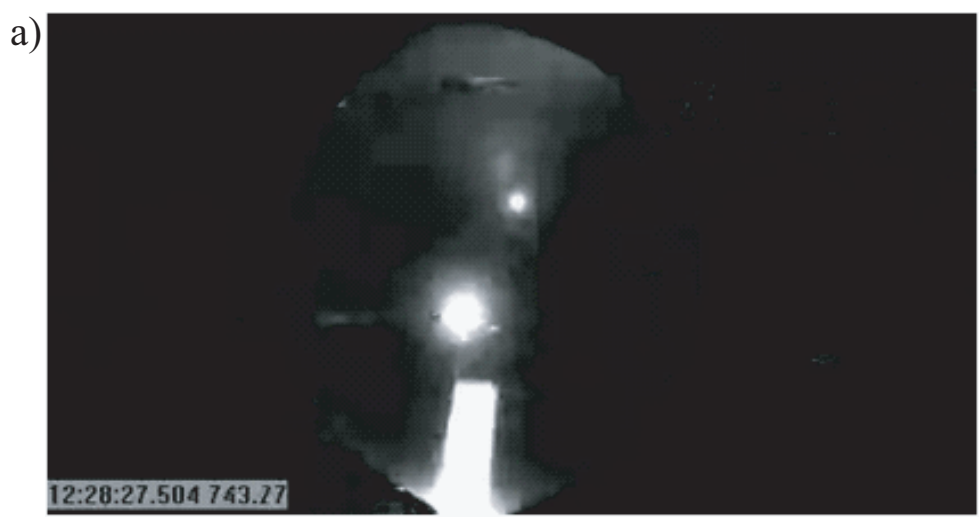

b)

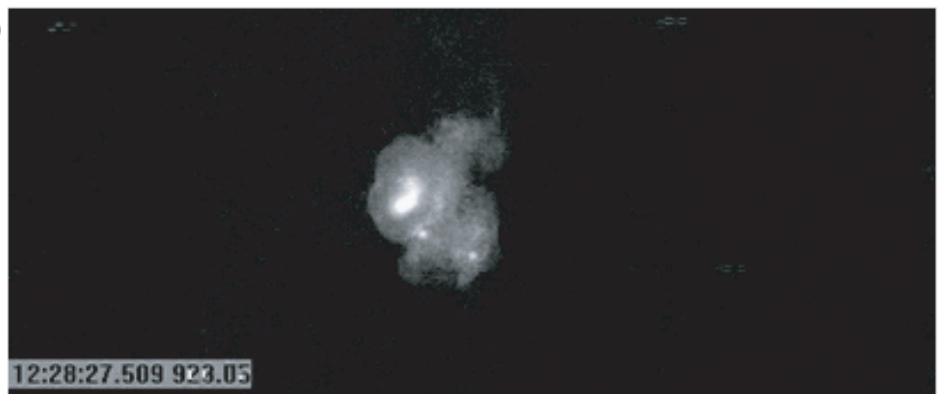

c)

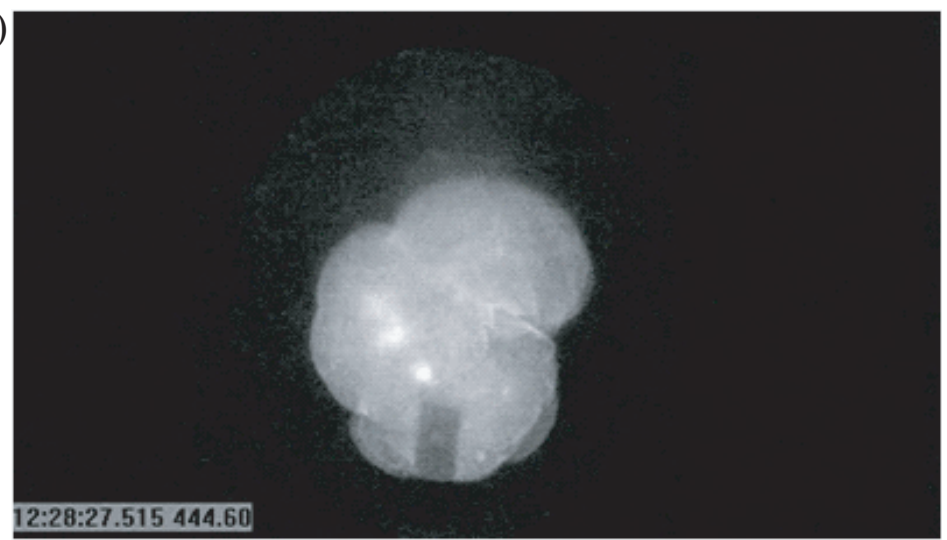

d)

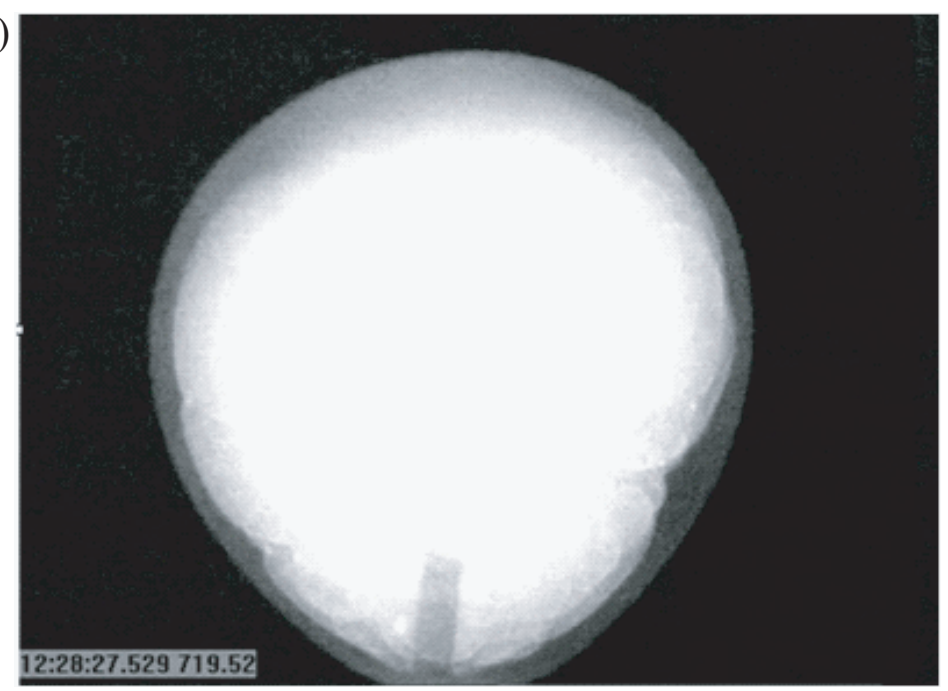

Figure 13. Ignition initiated by an attached discharge: discharge ignition (a); ignition of mixture (b); development of flame front (c); area of combustion (d)

Figure 15 shows the trajectory of balloon shell after detonation initiation for stoichiometric mixture $(\alpha=1)$ based on numerical calculations (solid line), theoretical predictions 


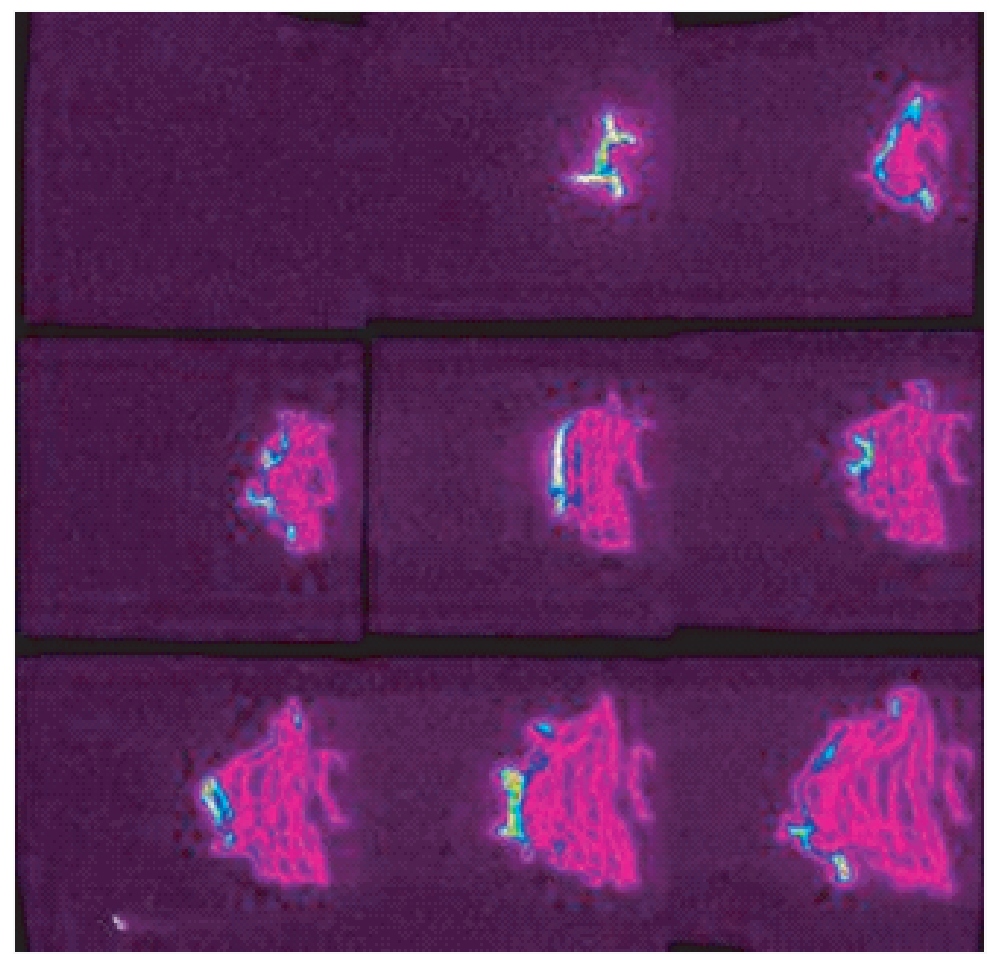

Figure 14. Speed camera frames of subcritical streamer discharge

(dashed line) and experimental measurements (bullets). Movement of the shell does not start immediately, it starts with some delay. The radiation associated with the reaction is terminated as soon as the detonation wave reaches the shell.

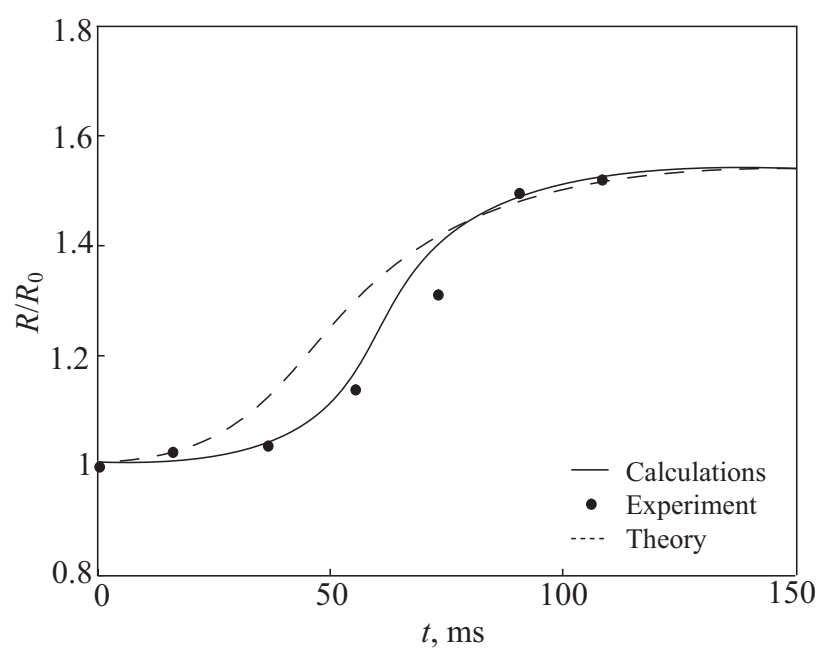

Figure 15. Time history of balloon radius for stoichiometric mixture

\section{Conclusion}

Ignition of the air-propane mixture by a sub-critical streamer discharge yields more than fourfold, from $1.5 \mathrm{~m} / \mathrm{s}$ to $7 \mathrm{~m} / \mathrm{s}$, increase in combustion speed compared to conventional spark ignition. An increase in combustion efficiency is also observed. Streamer discharge ignition of particularly poor air-fuel mixture with excess oxidant ratio greater than the 
flammability limit has been demonstrated under normal conditions. The indirect evidence suggests that the ignition by the microwave discharge is of the non-thermal nature. The advantages of igniting the fuel mixture by streamer discharge is attributed to the ultraviolet radiation emitted by oxygen atoms subjected to the discharge. The ultraviolet radiation generation causes formation of the non-equilibrium cold plasma with avalanche increase in the number of free electrons. The microwave streamer ignition can be considered for the application in internal combustion engines to replace the conventional spark ignition.

\section{Acknowledgements}

This work was financially supported by the Ministry of Education and Science of Russian Federation (agreement No 14.577.21.0277, unique identifier of applied scientific research RFMEFI57717X0277).

\section{References}

[1] Phylippov Yu.G., Dushin V.R., Nikitin V.F., Nerchenko V.A., Korolkova N.V., Guendugov V.M. Fluid mechanics of pulse detonation thrusters. Acta Astronautica, 2012, 76(1), 115-126.

[2] Smirnov N.N., Betelin V.B., Nikitin V.F., Phylippov Yu.G., Jaye Koo. Detonation engine fed by acetylene-oxygen mixture. Acta Astronautica, 2014, 104(1), 134-146.

[3] Kim H.H., Won S.H., Santner J., Chen Z., Ju Y. Measurements of the critical initiation radius and unsteady propagation of n-decane/air premixed flames. Proceedings of the Combustion Institute, 2013, 34(1), 929-936.

[4] Starikovskiy A., Aleksandrov N., Rakitin A. Plasma-assisted ignition and deflagrationto-detonation transition. Philosophical Transactions of the Royal Society of London, 2012, A370, 740-773.

[5] Beduneau J.-L., Kim B., Zimmer L., Ikeda Y. Measurements of minimum ignition energy in premixed laminar methane/air flow by using laser induced spark. Combustion and Flame, 2003, 132(4), 653-665.

[6] Do H., Im S., Cappelli M.A., Mungal M.G. Plasma assisted flame ignition of supersonic flows over a flat wall. Combustion and Flame, 2010, 157(12), 2298-2305.

[7] Michael J.B., Dogariu A., Shneider M.N., Miles R.B. Subcritical microwave coupling to femtosecond and picosecond laser ionization for localized, multipoint ignition of methane/air mixtures. Journal of Applied Physics, 2010, 108(9), 093308.

[8] Khodataev K.V. Numerical modeling of the combustion, assisted by the microwave undercritical discharge in supersonic flow. AIAA Paper, 2005, 2005-0985.

[9] Leonov S.B., Firsov A.A., Yarantsev D.A., Bolshov M.A., Kuritsyn Yu.A., Liger V.V., Mironenko V.R. Dynamics of $\mathrm{H}_{2} \mathrm{O}$ temperature and concentration in zone of plasmaassisted high-speed combustion. AIAA Paper, 2011, 2011-972.

[10] Napartovich A.P., Kochetov I.V., Leonov S.B. Calculation of the dynamics of ignition of an airhydrogen mixture by nonequilibrium discharge in a high-velocity flow. High Temperature, 2005, 43(5), 673-679.

[11] Starik A.M., Kozlov V.E., Titova N.S. On the influence of singlet oxygen molecules on the speed of flame propagation in methane-air mixture. Combustion and Flame, 2010, $157(2), 313-327$.

[12] Starikovskaia S.M. Plasma assisted ignition and combustion. Journal of Physics D: Applied Physics, 2006, 39(13), 265-299. 
[13] Popov N.A. The effect of nonequilibrium excitation on the ignition of hydrogen-oxygen mixtures. High Temperature, 2007, 45(2), 261-279.

[14] Leonov S.B., Yarantsev D.A. Plasma-induced ignition and plasma-assisted combustion in high-speed flow. Plasma Sources Science and Technology, 2007, 16(1), 132-139.

[15] Correale G., Rakitin A., Nikipelov A., Pancheshnyi S., Popov I., Starikovskiy A., Shiraishi T., Urushihara T., Boot M. Non-equilibrium plasma ignition for internal combustion engines. SAE Technical Paper, 2011, 2011-24-0090.

[16] Lefkowitz J.K., Guo P., Ombrello T., Won S.-H., Stevens C.A., Hoke J.L., Schauer F., Ju Y. Schlieren imaging and pulsed detonation engine testing of ignition by a nanosecond repetitively pulsed discharge. Combustion and Flame, 2015, 162(6), 2496-2507.

[17] Lefkowitz J.K., Ombrello T. Study of nanosecond pulsed high frequency discharge ignition in a flowing methane/air mixture. AIAA Paper, 2017, 2017-1777.

[18] Grachev L.P., Esakov I.I., Khodataev K.V. Features of the development of pulsed microwave discharges in various gases in a quasioptical beam. Technical Physics, 1998, 43(4), 378-381.

[19] Aleksandrov K.V., Grachev L.P., Esakov I.I., Khodataev K.V. Surface streamer microwave discharge. Technical Physics, 2002, 47(7), 851-855.

[20] Aleksandrov K.V., Grachev L.P., Esakov I.I., Fedorov V.V., Khodataev K.V. Domains of existence of various types of microwave discharge in quasi-optical electromagnetic beams. Technical Physics, 2006, 51(11), 1448-1456.

[21] Grachev L.P., Esakov I.I., Mishin G.I., Khodataev K.V. Front velocity of stimulated discharge in microwave beam. Journal of Technical Physics, 1995, 65(5), 21-30.

[22] Esakov I.I., Grachev L.P., Khodataev K.V., van Wie D.M. Efficiency of microwave discharges for propane ignition in cold high-speed airflows. AIAA Paper, 2005, 20050989.

[23] Khodataev K.V. The nature of surface MW discharges. AIAA Paper, 2010, 2010-1378.

[24] Bulat P.V., Volkov K.N. Model gas-dynamical problems possessing cylindrical and spherical symmetry and their solution with the aid of WENO schemes. Journal of Engineering Physics and Thermophysics, 2017, 89(2), 412-422.

[25] Volkov K. Numerical analysis of Navier-Stokes equations on unstructured meshes / Handbook on Navier-Stokes equations: theory and analysis. Nova Science, 2016, 365442 . 